\title{
An Iranian patient affected by autosomal recessive hypercholesterolemia due to a novel variant in the LDLRAP1 gene
}

\author{
Parisa Nikasa \\ Tabriz University of Medical Sciences \\ Bahareh Rabbani \\ Tehran University of Medical Sciences \\ Mohammad Saeid Hejazi \\ Tabriz University of Medical Sciences \\ Ata Firouzi \\ Iran University of Medical Sciences \\ Hossein Baharvand \\ Royan Institute for Stem Cell Biology and Technology \\ Mehdi Totonchi ( $\square$ totonchimehdi@gmail.com ) \\ Royan Institute for Stem Cell Biology and Technology \\ Nejat Mahdieh ( $\square$ nmahdieh@gmail.com ) \\ Iran University of Medical Sciences
}

\section{Case Report}

Keywords: Premature atherosclerotic cardiovascular disease, Autosomal recessive hypercholesterolemia, Rosuvastatin, Ezetimibe, LDLRAP1 gene

Posted Date: April 2nd, 2021

DOI: https://doi.org/10.21203/rs.3.rs-380832/v1

License: (a) (i) This work is licensed under a Creative Commons Attribution 4.0 International License. Read Full License

Version of Record: A version of this preprint was published at Clinical Pediatric Endocrinology on October 1st, 2021. See the published version at https://doi.org/10.1297/cpe.30.201. 


\section{Abstract}

Background: Autosomal recessive hypercholesterolemia (ARH) is a rare monogenic disorder resulting from mutations of the $L D L R A P 1$ gene, which leads to elevated LDL-C levels. Here, using whole exome sequencing (WES), we describe a 22-year-old Iranian female who carries a novel nonsense mutation in LDLRAP1.

Methods: Genetic investigations were performed for the patient and her family. She showed LDL-C level of $720 \mathrm{mg} / \mathrm{dL}$ since the age of 11 years. At the age of 13 years old, aortic valve repair surgery was performed due to severe aortic valve stenosis (AVS). At the age of 17, along with prescription of rosuvastatin plus ezetimibe, coronary angiography displayed the presence of serious stenotic lesions of the coronary arteries and also aortic valve, making the patient eligible for coronary artery bypass grafting (CABG) and aortic valve replacement (AVR).

Results: Genetic analysis showed the presence of a previously unreported homozygous $\angle D L R A P 1$ gene variant, c.649G $>T$, generating a nonsense mutation at amino acid 217, shortening the ARH protein from 308 to 217 amino acid, which removes AP-2 binding domain of $\mathrm{ARH}$, as an important part in LDL uptake.

Conclusion: During a 10-year treatment, we observed a 74\% reduction in LDL-C level. Despite the treatment with maximal dose of rosuvastatin plus ezetimibe, the results of coronary angiography demonstrated severe supravalvular aortic stenosis (SVAS) resulted in significant stenotic lesions of the coronary arteries and aortic valve. This highlights the importance of WES in early diagnosis of $\mathrm{ARH}$, and it is proposed to prevent or at least delay the onset of the cardiovascular events.

\section{Introduction}

Hypercholesterolemia is a fundamental risk factor for the development of atherosclerosis and cardiovascular disease (1). Familial hypercholesterolemia (FH), as a life-threatening monogenic disorder which is characterized by high levels of LDL-C, is classified into dominant and recessive types. The most common dominant form of $\mathrm{FH}$, which is commonly reported with a prevalence of 1 in 500 individuals, is cause0-d by mutations in the LDL receptor ( $L D L R)$ gene (MIM 606945) $(2,3)$. Elevated levels of plasma LDL-C in dominant form of FH may also result from mutations in the $A P O B$ gene (MIM 107730), coding the receptor-binding site of the apolipoprotein B-100 (Apo B-100) (4), and gain of function mutations in the PCSK9 gene (MIM 607786), which increase LDLRs degradation (5). Mutations in LDLR adopter protein 1 (LDLRAP1 also called ARH) (MIM 605747) cause autosomal recessive inheritance pattern of $\mathrm{FH}$ called $\mathrm{ARH}$, with the estimated prevalence of less than 1 in a population of 1,000,000. ARH (Online Mendelian Inheritance in Man [OMIM] catalog number 603813) is a rare monogenic disease caused by the loss-of-function mutations in the ARH protein (GenBank Accession no. NP_056442), encoded by LDLRAP1 gene (GenBank Accession no. NG_008932) (6). ARH has been considered as a phenocopy of homozygous familial hypercholesterolemia (HoFH; OMIM number 143890), so that most ARH patients are clinically indiscernible from HoFH (7). HoFH is a disease caused by two defective LDLR genes, with approximated prevalence of 6 individuals per million. The manifestations of ARH include severe hypercholesterolemia (typically, LDL-C >400 mg/dl), cutaneous xanthomas, bilateral corneal arcus, atheromatous involvement of the aortic valve, aortic stenosis and premature atherosclerotic cardiovascular disease (ASCVD) $(8,9)$. However, for reasons that are still to be elucidated, the ARH phenotype is more variable, generally have lower rates of ASCVD, and patients are more responsive to lipid-lowering treatment than HoFH patients (10, 11). Here in this report, we describe a novel variant, c.649G>T, p.Glu217Ter, in exon 7 of the LDLRAP1 in homozygous state, which to the best of our knowledge, has not been reported before, and also the patient's clinical response to long-term lipid-lowering treatment is discussed.

\section{Material And Methods}

Genetic counselling for a 17-year-old female patient with sever hypercholesterolemia was performed by our physicians group and a medical geneticist in our cardiovascular center in 2015. Informed consent was obtained for biochemical analysis as well as clinical and genetic studies. Genomic DNA was extracted from peripheral blood according to standard protocols (12). A gene panel-based next-generation sequencing (NGS) was performed to find casual variant(s) in the known involved genes of FH, including $L D L R$, $\angle D L R A P 1, P C S K 9$ and $A P O B$ (13). PCR primers were designed for the target region of $\angle D L R A P 1$ gene and the target region was amplified according to a conventional protocol. Afterward, to validate the novel variant, DNA sequencing of PCR products was performed using a BigDye termination method by sequencing analyzer of ABI3500XL model (PE Applied BioSystems, Massachusetts, USA). In silico analysis was performed to predict pathogenicity of the variant using MutationTaster (14), SIFT (15) and CADD (16).

Page 2/8 


\section{Results}

\subsection{Clinical report}

The patient is a 22-year-old female from a small village in llam, Iran. When she was 10 years old, the earliest clinical manifestations became apparent and she was referred to a physician due to the existence of tendon xanthomas on her hands, elbows and knees. She was aware of her severe hypercholesterolemia since she was 11 years old, with an LDL-C concentration of $720 \mathrm{mg} / \mathrm{dL}$. A daily pharmacological treatment of $4 \mathrm{~g}$ cholestyramine was initiated. The patient reported that she had undergone subaortic web resection and aortoplasty due to her uncontrolled hyperlipidemia in 2011 at the age of 13. She came to our cardiovascular center in Tehran in January 2015 for the study of her hypercholesterolemia. Bilateral corneal arcus, xanthomas and xanthelasmas were present. The plasma lipid profile was performed revealing severe hypercholesterolemia. Secondary causes of hypercholesterolemia, including renal diseases, diabetes mellitus and thyroid disease were ruled out. Liver enzymes levels were normal. Her physical examination revealed blood pressure of $110 / 70 \mathrm{mmHg}$ and body mass index (BMI) of $29 \mathrm{~kg} / \mathrm{m}^{2}$. At the age of 17 years, assessment of coronary arteries by coronary angiography revealed $50 \%$ ostial lesion of the left main artery and the right coronary artery was retrogradely filled by the left system. There was also a significant pressure gradient of $80 \mathrm{mmHg}$ in favor of significant AVS. On echocardiography, she had normal left ventricular systolic function and there was serial stenosis. The mitral and the tricuspid valves had mild regurgitation and the pulmonary artery pressure was normal. Therefore, although the patient was candidate for CABG and AVR, she rejected this surgery for personal reasons. Genetic analysis determined a homozygous $L D L R A P 1$ gene variant NM_015627.2: c.649G>T, NP_056442.2: p. Glu217Ter, which caused ARH disease. The other genes involved in FH including LDLR, PCSK9 and APOB didn't have any known pathologic mutation. A pharmacological treatment with rosuvastatin $(60 \mathrm{mg} /$ day) plus ezetimibe $(10 \mathrm{mg} /$ day $)$ was initiated. In May 2018, an angiography was performed and she was diagnosed with severe SVAS resulted in significant stenotic lesions of the coronary arteries and aortic valve. It was suggested that the patient should have CABG and AVR, but she refused to do so again. The family history displayed that the parents were cousins and had given birth four children (two males and two females), two of whom had passed away for unknown reasons. The lipid profile of the patient's mother (at the age of 44 years) was at normal range: TC$198 \mathrm{mg} / \mathrm{dL}, \mathrm{LDL}-\mathrm{C}-105 \mathrm{mg} / \mathrm{dL}, \mathrm{HDL}-\mathrm{C}-62 \mathrm{mg} / \mathrm{dL}$ and TG-150 mg/dL. The patient's father had died at the age of 42 years from coronary artery disease and his lipid profile is unavailable (Fig. 1).

\subsection{Laboratory Testing}

In January 2015, when the patient (at the age of 17 years) came to our cardiovascular center, the plasma lipid profile was performed, which showed severe hypercholesterolemia: total cholesterol (TC) $520 \mathrm{mg} / \mathrm{dL}$, low-density lipoproteins-cholesterol (LDL-C) 446 $\mathrm{mg} / \mathrm{dL}$, high-density lipoproteins-cholesterol (HDL-C) $57 \mathrm{mg} / \mathrm{dL}$ and triglycerides (TG) $93 \mathrm{mg} / \mathrm{dL}$. We started a pharmacological treatment with rosuvastatin $(60 \mathrm{mg} /$ day) plus ezetimibe $(10 \mathrm{mg} /$ day). The treatment process from January 2015 and the consequent lipid responses are shown in Table 1. Undergoing this treatment, the cutaneous xanthomas decreased markedly and also a remarkable reduction of plasma LDL-C was determined (from $402.5 \pm 31.1$ to $103.8 \pm 26.02 \mathrm{mg} / \mathrm{dL}$ ) associated with a significant increase in alanine aminotransferase (ALT, from $16.25 \pm 6.05$ to $49.2 \pm 25.2 \mathrm{UI} / \mathrm{L}$ ). The treatment produced no significant side effects. Table 1 shows the changes in lipid levels obtained in baseline situation (mean of 4 determinations) and along cholesterol-lowering treatment (mean of monthly determinations).

Table 1. Evolution of lipid profile of the patient before and during treatment with rosuvastatin ( $60 \mathrm{mg} /$ day) plus ezetimibe (10 $\mathrm{mg} /$ day) 


\begin{tabular}{|lllll|}
\hline Plasma parameter & Before drugs & R60 + E10 & Percent change & $P^{*}$ \\
\hline TC mg/dL & $482.25 \pm 28.4$ & $169.7 \pm 32.1$ & -64.8 & $<.0001$ \\
\hline LDL-C mg/dL & $402.5 \pm 31.1$ & $103.8 \pm 26.02$ & -74.2 & $<.001$ \\
\hline HDL-C mg/dL & $61.5 \pm 5.4$ & $48.1 \pm 6.8$ & -21.8 & $<.01$ \\
\hline TG mg/dL & $81.5 \pm 13.6$ & $62.8 \pm 11.5$ & -22.9 & $<.052$ \\
\hline
\end{tabular}

TC, total cholesterol; LDL-C, LDL-cholesterol; HDL-C, HDL-cholesterol; TG, triglycerides.

Values are mean \pm SD. *Comparisons were performed by student's test for paired data.

\subsection{Genetic Analysis}

The results of NGS analysis did not identify any pathogenic changes in $L D L R, P C S K 9$ and $A P O B$. However, a novel homozygous variant, c. $649 \mathrm{G}>\mathrm{T}$, in the $\angle D L R A P 1$ gene, was detected in the patient. The identified mutation c.649G $>\mathrm{T}$ leads to the formation of stop codon at amino acid residue 217 of ARH protein (p. Glu217Ter). Sanger sequencing was used to validate the presence of the new variant identified by NGS (Fig. 2). The same mutation c.649G>T was identified in the $\angle D L R A P 1$ in heterozygosity for her mother and living brother. According to the American College of Medical Genetics and Genomics (ACMG) guideline 2015 (17), this nonsense variant can be assumed to disrupt the function of protein using nonsense-mediated decay of an altered transcript. However, in silico analysis were performed by available software tools, including CADD (Combined Annotation Dependent Depletion), SIFT and MutationTaster to predict pathogenicity of the variant. The results of the analysis predicted this variant as damaging due to a stop codon mutation in exon 7 of the LDLRAP1 (Table 2). Furthermore, the sequences were mapped to the GRCh37/hg19 human reference sequence. Data bases used for assessment were Online Mendelian Inheritance in Man (www.omim.org); Human Gene Mutation Database (HGMD $®$ Public) (www.biobaseinternational.com/hgmd) from BIOBASE Corporation and Gene Tests (www.genetests.org).

These software tools predicted the pathogenic effect of the variant. The identified nonsense variant was absent in HGMD, dbSNP version 147, ClinVar databases, Iranome and Exome Sequencing Project (ESP). This variant was not found in the literature as well. The novel variant was named c.649G>T, based on Human Genome Variation Society (HGVS) nomenclature standards and determined based on NM_015627.2 and NP_056442.2. The sequence variant was submitted to ClinVar database.

Table 2. Several online databases that used to predict the pathogenicity of c.649G>T, p.Glu217Ter in the family. $\rrbracket$

\begin{tabular}{|c|c|c|c|c|c|c|c|c|c|}
\hline Gene & NM & NP & Exon & & Alteration & In silico analysis & ools & & $\mathrm{dbSNP}$ \\
\hline \multirow[t]{2}{*}{$\angle D L R A P 1$} & NM_015627 & NP_056442 & 7 & $\begin{array}{l}\text { Nucleic } \\
\text { Acid }\end{array}$ & Amino Acid & MutationTaster & CADD & SIFT & novel \\
\hline & & & & c. $649 \mathrm{G}>\mathrm{T}$ & p.Glu217Ter & $\begin{array}{l}\text { Disease } \\
\text { causing }\end{array}$ & 42 & Damaging & \\
\hline
\end{tabular}

\section{Discussion}

Different mutations in $L D L R A P 1$ gene, which lead to ARH disease, have been reported (8). According to currently available information in the Human Gene Mutation Database (HGMD; http://www.hgmd.cf.ac.uk/ac/gene.php?gene=LDLRAP1), 26 pathogenic variants in the coding sequence of $\angle D L R A P 1$ have been reported. Here, in this clinical report, we describe a novel variant c. $649 \mathrm{G}>\mathrm{T}$, $\mathrm{p}$. Glu217Ter in the LDLRAP1 gene in a homozygous form. 
The human $\angle D L R A P 1$ gene is comprised of nine exons, located on the short arm of chromosome 1 (1p36.11) and encodes ARH protein with 308 amino acids. ARH binds to LDLR and mediates its internalization through clathrin-coat machinery. ARH undertakes this by simultaneously binding of its N-terminal phosphotyrosine-binding domain (PTB: amino acid 48-175) to the FDNPXY motif within the cytoplasmic tail of LDLR, Clathrin Box module (C; LLDLE motif: amino acid 212-216) to clathrin proteins, and an AP-2binding module (AP2: amino acid 248-279) within its C-terminul to the $\beta 2$-adaptin subunit of AP-2 (18). As the ARH proband (Fig. 1) was homozygous for a nonsense mutation in codon 217, the translation can be stopped just outside of the Clathrin Box domain. Due to this mutation, the LDL-uptake by the hepatocytes can be disrupted and consequently LDL-C levels in the plasma increases.

Previous studies indicated that the ARH phenotype is much milder than that of LDLR-HoFH (10). However, recently the finding of a worldwide collaboration project studying $52 \mathrm{ARH}$ patients (28 females, 24 males) during a mean follow-up of $14.1 \pm 7.3$ years showed that incidence rate of ASCVD in the treated ARH patients was comparable to the patients with HoFH (19). Another similar phenotype with LDLR-HoFH is the risk of AVS, which occurs relatively often in patients with ARH (19). SVAS is a rare finding in ARH patients with aortic stenosis (20), which is typically seen in LDLR-HoFH. In agreement with previous studies $(19,21)$, the clinical manifestation of our ARH patient mimicked the most severe form of genetic hypercholesterolemia, LDLR-HoFH: cutaneous xanthomas found at early age (10 years), the levels of LDL-C between LDL receptor-negative and receptor-defective HoFH, and also AVS and SVAS were present at the time of writing this report (22-year-old).

Lipid-lowering response of patients with ARH is much better than those with LDLR-HoFH (22), specifically LDLR-negative ones. The greater responsiveness to statins in ARH compared with $\mathrm{FH}$, may be illuminated by the following observations: the comparison rate of very-low density lipoprotein (VLDL) clearance in Arh-/- and Ldlr-/- mice was described by Jones et al (23); their experiments showed that ARH is not required for LDLR-dependent uptake of VLDL by the liver, which can cause the preservation of VLDL remnants clearance and reduction of the production rate of LDL from VLDL; this result was recently confirmed by kinetic studies in Japanese patients with $\mathrm{ARH}$, which documented increased remnant lipoprotein fraction clearance, and a decreased conversion of VLDL to LDL (24). However, there are inadequate indications for clinical management and cardiovascular outcomes of ARH patients (19). Considerably, in spite of treatment with high dose of rosuvastatin plus the cholesterol absorption inhibitor ezetimibe, our patient presented a severe cardiovascular involvement, AVS and SVAS at a very early age (21-year-old). The previous studies in ARH patients show great variability in $L D L-C$ lowering response to statin treatment, as in some ARH patients, the treatment with maximal dose of statin associated with ezetimibe induces an LDL-C decrease by $80 \%$ to $90 \%$, whereas the others in spite of maximum therapy do not reach the $20 \%$ reduction in LDL-C levels $(20,25)$. In our ARH patient, the $74 \%$ reduction of plasma LDL-C concentration was achieved through a high dose of rosuvastatin plus ezetimibe. Proprotein convertase subtilisin/kexin type 9 (PCSK9) inhibitor monoclonal antibodies, alirocumab and evolocumab, which are recently developed lipid-lowering drugs, can regulate the level of LDLR through triggering its degradation (26). The findings of very few available results of the treatment with PCSK9 inhibitors indicate extensively variable responses in treated ARH patients, LDL-C reduction ranging from none to $40 \%$ after up to 12 weeks $(20,27)$. Our patient was not taking the PCSK9 inhibitors because of economic reasons. Despite the availability of several pharmacological therapies, the level of LDL-C in ARH patients remains far from target levels, which can cause a poor long-term cardiovascular prognosis. Current guidelines for FH patients recommend a LDL-C goal of $<100 \mathrm{mg} / \mathrm{dL}$ for adults or $<70 \mathrm{mg} / \mathrm{dL}$ for adults with coronary heart diseases $(\mathrm{CHD})$ or diabetes, and $<135 \mathrm{mg} / \mathrm{dL}$ for children (28). Although we couldn't reach the LDL-C goal ( $<70 \mathrm{mg} / \mathrm{dL}$ for adults with $\mathrm{CHD})$, however, we observed $74 \%$ (from $402.5 \pm 31.1$ to $103.8 \pm 26.0 \mathrm{mg} / \mathrm{dL}$ ) treatment-mediated reduction in LDL-C level in our patient. This is a remarkable response to the therapy and decrease in LDL-C level, implying the patient's sensitivity to the treatment.

In conclusion, our data highlight the importance of an early identification of ARH patients and appropriate treatment to prevent or at least delay the onset of cardiovascular events, and approve the fact that an efficient treatment protocol for ARH is still lacking (19, 28). Additionally, our clinical report represents a novel nonsense G>T mutation at position 649 causing ARH. In our patient, the ARH was manifested with SVAS resulted in significant stenotic lesions of the coronary arteries and aortic valve.

\section{Declarations}

The authors declare no conflicts of interest.

\section{Acknowledgments}

We would like to thank the patient and her family for their willing participation and cooperation with us and we also especially thank Tine Tricot (SCIL, KU Leuven, Belgium), Mohammad Mostafa Ansari Ramandi (Heart Valve Disease Research Center, University of 
Medical Sciences, Tehran, Iran) for providing critical comments in revising the article and personnel of Cardiogenetic Research Center, Rajaie Cardiovascular, Medical, and Research Center for technical assistance. This work was supported in part by a research grant to Dr. Mahdieh from Iran's National Science Foundation (98023504) and the Research Deputyship of Iran University of Medical Sciences (98-3-37-16373).

\section{CRediT authorship contribution statement}

Parisa Nikasa: Collection of all the clinical data of the patient, Formal analysis, Writing-original draft. Bahareh Rabbani: Methodology, Writing-review \& editing. Mohammad saeid Hejazi: Discussion, Writing-review \& editing. Ata Firouzi: Data curation, Writing- review \& editing. Hossein Baharvand: Consultation, Writing- review \& editing. Mehdi Totonchi: Supervision, Writing - review \& editing. Nejat Mahdieh: Conceptualization, Methodology, Validation, Writing - review \& editing.

\section{References}

1. Sharrett AR, Ballantyne C, Coady S, Heiss G, Sorlie P, Catellier D, Patsch W. Coronary heart disease prediction from lipoprotein cholesterol levels, triglycerides, lipoprotein (a), apolipoproteins Al and B, and HDL density subfractions: The Atherosclerosis Risk in Communities (ARIC) Study. Circulation. 2001; 104(10):1108-1113.

2. Brown MS, Goldstein JL. A receptor-mediated pathway for cholesterol homeostasis. Science. 1986; 232(4746):34-47.

3. de Ferranti SD, Rodday AM, Mendelson MM, Wong JB, Leslie LK, Sheldrick RC. Prevalence of familial hypercholesterolemia in the 1999 to 2012 United States national health and nutrition examination surveys (NHANES). Circulation. 2016; 133(11):1067-1072.

4. Innerarity TL, Mahley RW, Weisgraber KH, Bersot TP, Krauss RM, Vega GL, Grundy SM, Friedl W, Davignon J, McCarthy BJ. Familial defective apolipoprotein B-100: a mutation of apolipoprotein B that causes hypercholesterolemia. J Lipid Res. 1990; 31(8):1337-1349.

5. Abifadel M, Varret M, Rabès JP, Allard D, Ouguerram K, Devillers M, Cruaud C, Benjannet S, Wickham L, Erlich D, Derré A, Villéger L, Farnier M, Beucler I, Bruckert E, Chambaz J, Chanu B, Lecerf JM, Luc G, Moulin P, Weissenbach J, Prat A, Krempf M, Junien C, Seidah NG, Boileau C. Mutations in PCSK9 cause autosomal dominant hypercholesterolemia. Nat Genet. 2003; 34(2):154-156.

6. Garcia CK, Wilund K, Arca M, Zuliani G, Fellin R, Maioli M, Calandra S, Bertolini S, Cossu F, Grishin N, Barnes R, Cohen JC, Hobbs $\mathrm{HH}$. Autosomal recessive hypercholesterolemia caused by mutations in a putative LDL receptor adaptor protein. Science. 2001; 292(5520):1394-1398.

7. Ito MK, Watts GF. Challenges in the diagnosis and treatment of homozygous familial hypercholesterolemia. Drugs. 2015; 75(15):1715-1724.

8. Fellin R, Arca M, Zuliani G, Calandra S, Bertolini S. The history of Autosomal Recessive Hypercholesterolemia (ARH). From clinical observations to gene identification. Gene. 2015; 555(1):23-32.

9. Tada H, Kawashiri M-a, Nohara A, Inazu A, Kobayashi J, Mabuchi H, Yamagishi M. Autosomal recessive hypercholesterolemia: a mild phenotype of familial hypercholesterolemia: insight from the kinetic study using stable isotope and animal studies. J Atheroscler Thromb. 2015; 22(1):1-9.

10. Pisciotta L, Oliva CP, Pes GM, Di Scala L, Bellocchio A, Fresa R, Cantafora A, Arca M, Calandra S, Bertolini S. Autosomal recessive hypercholesterolemia (ARH) and homozygous familial hypercholesterolemia ( $\mathrm{FH})$ : a phenotypic comparison. Atherosclerosis. 2006; 188(2):398-405.

11. Sánchez-Hernández RM, Prieto-Matos P, Civeira F, Lafuente EE, Vargas MF, Real JT, Goicoechea FG, Fuentes FJ, Pocovi M, Boronat M, Wägner AM, Masana L. Autosomal recessive hypercholesterolemia in Spain. Atherosclerosis. 2018; 269:1-5.

12. Green MR, Sambrook J. Molecular cloning: a laboratory manual. Cold Spring Harbor Laboratory Press, Cold Spring Harbor, New York, 2012; pp 1-71.

13. Rodríguez-Jiménez C, Gómez-Coronado D, Vargas MF, Cerrato F, Lahoz C, Saban-Ruiz J, Gonzalez-Nieto D, Lasunción MA, Mostaza JM, Rodríguez-Nóvoa S. A new variant (c. 1A>G) in LDLRAP1 causing autosomal recessive hypercholesterolemia: Characterization of the defect and response to PCSK9 inhibition. Atherosclerosis. 2019; 284:223-229.

14. Schwarz JM, Cooper DN, Schuelke M, Seelow D. MutationTaster2: mutation prediction for the deep-sequencing age. Nat Methods. 2014; 11(4):361-362. 
15. Kumar P, Henikoff S, Ng PC. Predicting the effects of coding non-synonymous variants on protein function using the SIFT algorithm. Nat Protoc. 2009; 4(7):1073-1081.

16. Kircher M, Witten DM, Jain P, O'Roak BJ, Cooper GM, Shendure J. A general framework for estimating the relative pathogenicity of human genetic variants. Nat Genet. 2014; 46(3):310-315.

17. Richards S, Aziz N, Bale S, Bick D, Das S, Gastier-Foster J, Grody WW, Hegde M, Lyon E, Spector E, Voelkerding K, Rehm HL. Standards and guidelines for the interpretation of sequence variants: a joint consensus recommendation of the American College of Medical Genetics and Genomics and the Association for Molecular Pathology. Genet Med. 2015; 17(5):405-423.

18. Zhao Z, Pompey S, Dong H, Weng J, Garuti R, Michaely P. S-nitrosylation of ARH is required for LDL uptake by the LDL receptor. J Lipid Res. 2013; 54(6):1550-1559.

19. D’Erasmo L, Minicocci I, Nicolucci A, Pintus P, Roeters Van Lennep JE, Masana L, Mata P, Sánchez-Hernández RM, Prieto-Matos P, Real JT, Ascaso JF, Lafuente EE, Pocovi M, Fuentes FJ, Muntoni S, Bertolini S, Sirtori C, Calabresi L, Pavanello C, Averna M, Cefalù AB, Noto D, Pacifico AA, Pes GM, Harada-Shiba M, Manzato E, Zambon S, Zambon A, Vogt A, Scardapane M, Sjouke B, Fellin R, Arca M. Autosomal recessive hypercholesterolemia: long-term cardiovascular outcomes. J Am Coll Cardiol. 2018; 71(3):279-288.

20. Vaverkova H, Tichy L, Karasek D, Freiberger T. A case of autosomal recessive hypercholesterolemia caused by a new variant in the LDL receptor adaptor protein 1 gene. J Clin Lipidol. 2019; 13(3):405-410.

21. Soutar AK, Naoumova RP, Traub LM. Genetics, clinical phenotype, and molecular cell biology of autosomal recessive hypercholesterolemia. Arterioscler Thromb Vasc Biol. 2003; 23(11):1963-1970.

22. Gidding SS, Champagne MA, de Ferranti SD, Defesche J, Ito MK, Knowles JW, McCrindle B, Raal F, Rader D, Santos RD, LopesVirella M, Watts GF, Wierzbicki AS. The agenda for familial hypercholesterolemia: a scientific statement from the American Heart Association. Circulation. 2015; 132(22):2167-2192.

23. Jones C, Garuti R, Michaely P, Li W-P, Maeda N, Cohen JC, Herz J, Hobbs HH. Disruption of LDL but not VLDL clearance in autosomal recessive hypercholesterolemia. J Clin Invest. 2007; 117(1):165-174.

24. Tada H, Kawashiri M-a, Ikewaki K, Terao Y, Noguchi T, Nakanishi C, Tsuchida M, Takata M, Miwa K, Konno T, Hayashi K, Nohara A, Inazu A, Kobayashi J, Mabuchi H, Yamagishi M. Altered metabolism of low-density lipoprotein and very-low-density lipoprotein remnant in autosomal recessive hypercholesterolemia: results from stable isotope kinetic study in vivo. Circ Cardiovasc Genet. 2012; 5(1):35-41.

25. Muntoni S, Pisciotta L, Muntoni S, Bertolini S. Pharmacological treatment of a Sardinian patient affected by autosomal recessive hypercholesterolemia (ARH). J Clin Lipidol. 2015; 9(1):103-106.

26. Pećin I, Hartgers ML, Hovingh GK, Dent R, Reiner Ž. Prevention of cardiovascular disease in patients with familial hypercholesterolaemia: The role of PCSK9 inhibitors. Eur J Prev Cardiol. 2017; 24(13):1383-1401.

27. Saeed A, Virani SS, Jones PH, Ballantyne CM, Nambi V. Case reports of proprotein convertase subtilisin/kexin type 9 (PCSK9) inhibition nonresponse. J Clin Lipidol. 2018; 12(5):1141-1145.

28. Raal FJ, Hovingh GK, Catapano AL. Familial hypercholesterolemia treatments: guidelines and new therapies. Atherosclerosis. 2018; 277:483-492.

\section{Figures}




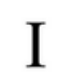

II

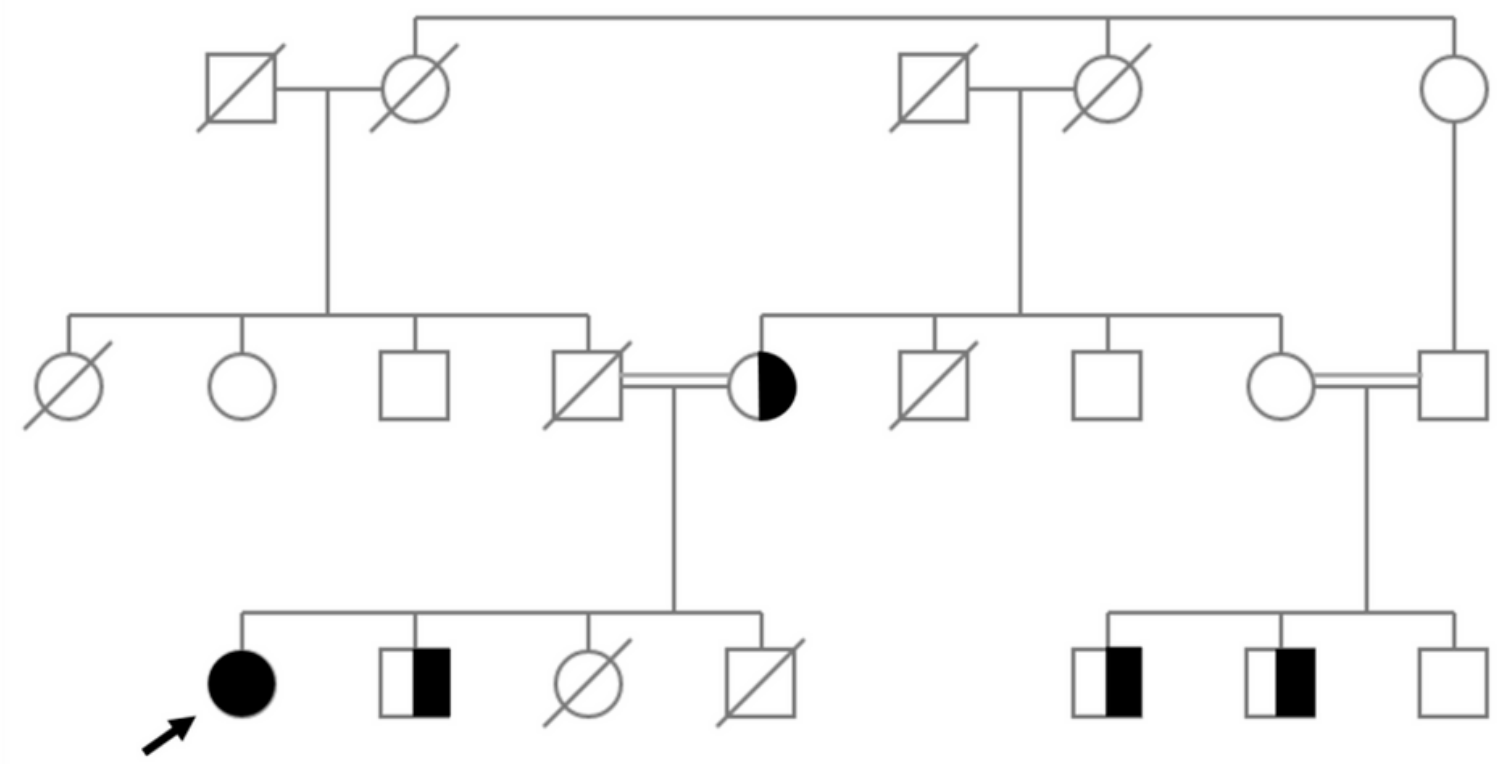

\section{Figure 1}

Pedigree of the family with ARH. The proband, which is indicated by black arrow was born to consanguineous parents (first cousins). Roman numerals show generation. Squares represent male and circles female subjects. Solid black symbol represents homozygote $\mathrm{ARH}$, half-black symbol represent heterozygote and crossed out symbol, deceased.

$\begin{array}{llllllllllllllllllllllllll} & G & G & A & C & I & I & A & G & A & G & G & A & G & A & C & A & G & C & I & A & A & G & G & \text { Reference sequence }\end{array}$

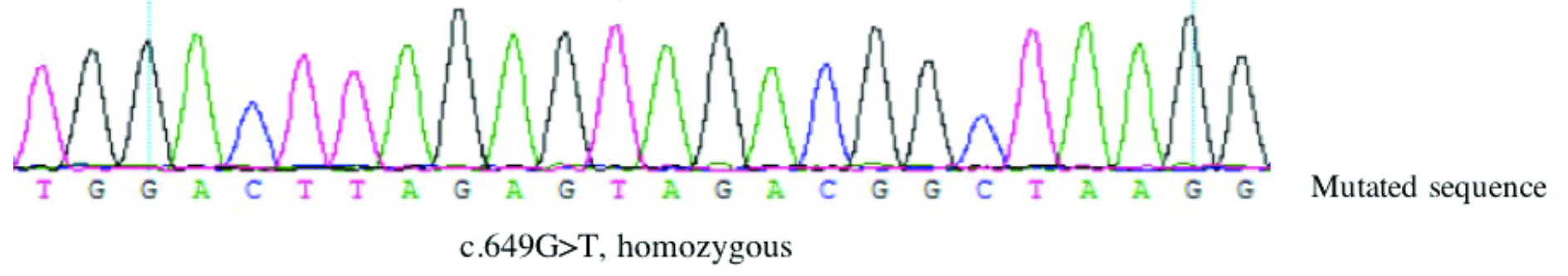

Figure 2

LDLRAP1 variant in an individual with ARH. Genomic sequencing showing the variant in LDLRAP1 in a homozygous state in the affected individual. 